\title{
Genetic background affects induced pluripotent stem cell generation
}

\author{
Lauren V Schnabel ${ }^{1}$, Christian M Abratte ${ }^{2}$, John C Schimenti ${ }^{2}$, Teresa L Southard ${ }^{2}$ and Lisa A Fortier ${ }^{1 *}$
}

\begin{abstract}
Introduction: The influence of genetic background on the ability to generate induced pluripotent stem cells (iPSCs) has the potential to impact future applications, but has yet to be examined in detail. The purpose of this study was to determine if genetic background affects the efficiency of generating iPSCs during early reprograming as well as the pluripotent stability of the iPSCs during later stages of reprograming.

Methods: Mouse embryonic fibroblasts (MEFs) were isolated from six strains of mice (NON/Lt; C57BL/6J; DBA/2J; BALB/CJ; 129S1/SvImJ; (CAST/EiJ) that were selected based on genetic diversity and differences in ability to produce embryonic stem cell (ESC) lines. MEFs were reprogramed via doxycycline-inducible lentiviral transduction of murine Oct4, KIf4, Sox2, and c-Myc. Differences in efficiency to generate iPSCs were assessed by comparing the total number of colonies, the percentage of colonies positive for alkaline phosphatase staining and the percentage of cells positive for SSEA1. IPSC colonies were expanded to establish doxycycline-independent cell lines whose pluripotency was then evaluated via ability to form teratomas in NOD.CB17-Prkdc scid/J mice. Proliferation of non-transduced parent MEFs from each strain was also examined over ten days under conditions that simulated reprograming.

Results: NON/LtJ and CAST/EiJ strains were more efficient than other strains in generating iPSCs for all parameters measured and parent MEFs from these strains were more proliferative than those from other strains. Doxycyclineindependent IPSC lines were established using standard conditions for all strains except BALB/CJ, which required a higher concentration (5x) of leukemia inhibitory factor (LIF). iPSCs from all strains were capable of producing teratomas in NOD.CB17-Prkdc scid/J mice.

Conclusions: The results of this study suggest that genetic background does affect iPSC generation and pluripotent stability. In addition, our results demonstrate that strain differences in efficiency to generate iPSCs during the early stages of reprograming are correlated with those observed in proliferation of parent MEFs. These findings have important implications both for future iPSC applications as well as for future investigation into determining the genes responsible for reprograming efficiency and stability.
\end{abstract}

\section{Introduction}

The induced pluripotent stem cell (iPSC) field continues to make rapid advances in terms of optimizing reprograming methods to circumvent clinical safety issues and characterization of the genetic and epigenetic composition of established iPSC lines [1-4]. The influence of genetic background on the ability to generate iPSCs, as well as the stability and quality of derived iPSCs for downstream applications, also has the potential to impact the future applications. However, the role of genetic

\footnotetext{
* Correspondence: laf4@cornell.edu

'Department of Clinical Sciences, College of Veterinary Medicine, Cornell University, Ithaca, NY 14853, USA

Full list of author information is available at the end of the article
}

background has yet to be examined in significant detail. The effect of genetic background on pluripotency has precedence in mice; it is well documented that there are dramatic strain differences in ability to produce embryonic stem cell (ESC) lines [5-8].

Many of the mouse iPSC studies to date have used mouse embryonic fibroblasts (MEFs) from transgenic mice of an undefined or hybrid background [9], or have used MEFs or tail tip fibroblasts (TTFs) derived from animals originally produced from hybrid ESCs [9-16]. Few studies have used MEFs or TTFs from a pure inbred strain [17-19]. To our knowledge, only one study to date has directly compared the ability of two different inbred strains to generate iPSCs [17]. In this study, Hanna et al.

\section{Biomed Central}


found that MEFs from NOD/ShiLt mice, a strain previously considered nonpermissive for ESC derivation, were capable of generating iPSCs, but that these iPSCs were dependent on exogenous transgene expression unlike the iPSCs derived from control 129Sv/Jae MEFs [17]. The authors determined that the NOD/ShiLtJ iPSCs were dependent upon ectopic expression of either KLF4 or c-MYC using constitutive lentiviruses, and that the cells were able to overcome this factor dependence when cultured in media supplemented with any of the following proteins or small molecules: WNT3a, which promotes iPSC derivation in the absence of c-MYC [20]; CHIR99021, a GSK3b inhibitor; or Kenpaullone, a GSK3b and CDK1/cyclin B inhibitor which has been shown to replace KLF4 during iPSC reprograming $[17,21]$. As the authors concluded, these results suggest that genetic background can affect the pluripotent stability of iPSCs and that reprograming and culture conditions may have to be modified for certain strains [17].

The purpose of this study was to determine if genetic background affects the efficiency of generating iPSCs during early reprograming as well as the pluripotent stability of the iPSCs during later stages of reprograming. We chose six different inbred strains of mice to examine, based on their genetic diversity [22-24] and on their differences in ability to produce ESC lines [5-8]. These six strains included five classical laboratory strains (NON/Lt), C57BL/6J, DBA/2J, BALB/cJ, and 129S1/SvlmJ) and one wild-derived inbred strain (CAST/EiJ) (Figure 1). Because 129-derived substrains such as $129 \mathrm{~S} 1 / \mathrm{Svlm}$ J support facile ESC line derivation [5-8], while both C57BL/6J and BALB/ cJ mice do not $[5,8]$, we reasoned that these strains would be useful for assessing potential differences in reprograming efficiency. In addition, three of the strains (C57BL/6), 129S1/SvlmJ, and CAST/EiJ) are progenitors of the Collaborative Cross that is proving effective for analyzing complex genetic phenotypes $[25,26]$. Knowledge on the potential differences between these strains in their ability to generate iPSCs and their pluripotent stability might therefore be amenable to genetic analysis.

In this study, we show that iPSC lines can be generated from all six of the strains examined using a lentiviral reprograming system [27-29] and that these cell lines are capable of forming teratomas in NOD.CB17-Prkdc $c^{\text {scid } / \mathrm{J}}$ mice. We demonstrate, however, that there are strain differences in efficiency of iPSC induction, growth, and maintenance requirements, and that these strain differences correlate with proliferative ability of the parental MEFs.

\section{Materials and methods}

Mice

Male and female mice from all six strains examined (NON/Ltt; C57BL/6J; DBA/2J; BALB/cJ; 129S1/SvlmJ; CAST/EiJ ) were purchased from The Jackson
Laboratory (Bar Harbor, ME, USA). For each strain, breeder trios were established for timed matings such that embryonic day-13.5 embryos could be collected and processed to generate MEFs. NOD.CB17-Prkdc scid/J mice, used for teratoma formation assays, were also purchased from The Jackson Laboratory. The use of mice in this study was approved by the Institutional Animal Care and Use Committee of Cornell University.

\section{MEF culture}

Embryonic day-13.5 embryos were isolated from the uteri of pregnant mice, lavaged with phosphate buffered saline (PBS), and eviscerated. Each embryo was then gently homogenized in MEF media comprising high glucose Dulbecco's modified eagle's medium (DMEM) containing $10 \%$ fetal bovine serum (FBS), penicillin (100 units $/ \mathrm{mL}$ ), and streptomycin (100 units/mL) and the resultant cell suspension was transferred to a $100-\mathrm{mm}$ tissue culture plate and incubated at $5 \% \mathrm{CO}_{2}, 90 \%$ humidity, and $37^{\circ} \mathrm{C}$. The plates were washed with PBS and the media changed daily until the MEFs were confluent, at which time they were trypsinized, resuspended in freeze media comprising DMEM with 10\% FBS and 10\% dimethyl sulfoxide (DMSO), and cryopreserved until further use. All experiments were performed using MEFs derived from two different embryos for each strain.

\section{Lentiviral constructs}

Lentiviral vectors for doxycycline-inducible transgene expression were constructed as previously described [27-29] using an FUW-based plasmid with a tetracycline operator (TetO) and a constitutive cytomegalovirus (CMV) promoter. Briefly, the viral packaging plasmids psPAX2 and pMD2.G (Addgene 12260 and 12259, Cambridge, MA, USA) as well as the plasmids encoding the reverse tetracycline transactivator (M2rtTA; Addgene 20342, Cambridge, MA, USA) and the mouse factors Oct4, Sox2, Klf4 and c-Myc (Addgene 20323, 20326, 20322 and 20324 respectively, also Cambridge) were purified from bacterial cultures. The vectors were then prepared by co-transfecting the viral packaging plasmids with plasmids encoding the reverse tetracycline transactivator and the reprograming factors into $293 \mathrm{~T}$ cells using the FuGENE ${ }^{\circledR} 6$ Transfection Reagent (Roche Applied Science, Indianapolis, IN, USA). Viral supernatants were collected at 48 and 72 hours, concentrated using an Amicon Ultra-15 centrifugal filter unit with an Ultracel-30 membrane (Millipore, Billerica, MA, USA), filtered through a 0.45 um filter, and stored in liquid nitrogen until used.

\section{Reprograming of MEFs and iPSC culture}

Passage 2 (P2) MEFs from each strain were seeded onto gelatin-coated tissue culture plates at a density of $6.75 \times$ $10^{3}$ cells $/ \mathrm{cm}^{2}$ in MEF media and allowed to adhere for 


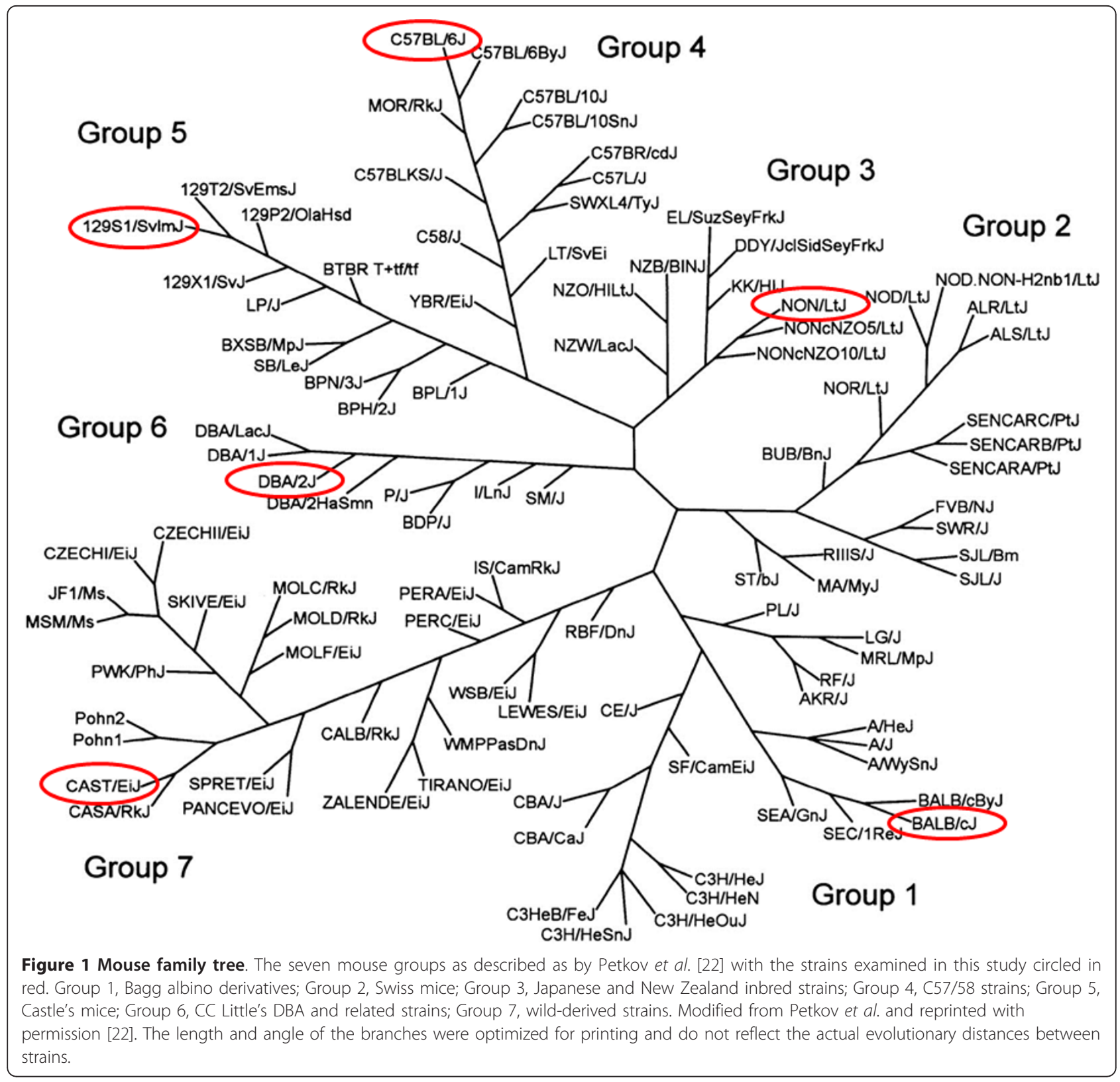

24 hours [27]. The culture media was then replaced with fresh MEF media supplemented with the viral supernatant described above. Following 24 hours of incubation with the viral supernatant, the culture media was changed to ESC media (KnockOut ${ }^{\mathrm{TM}}$ DMEM (Gibco, Grand Island, NY, USA) supplemented with 15\% KnockOut ${ }^{\mathrm{TM}}$ Serum Replacement (Gibco), recombinant LIF, MEM non-essential amino acids solution (100 $\mu \mathrm{m}), 2 \mathrm{mM}$ GlutaMAX ${ }^{\mathrm{TM}}$ (Gibco), $0.1 \mathrm{mM}$ 2-mercaptoethanol, penicillin (100 units $/ \mathrm{mL}$ ), streptomycin (100 units $/ \mathrm{mL})$, and doxycycline $(2 \mu \mathrm{g} / \mathrm{mL}$; Sigma, St. Louis, MO, USA)). Cells destined for flow cytometric analysis and for expansion were kept on original 6-well plates while cells destined for alkaline phosphatase (AP) staining and colony counting were trypsinized and passaged onto gelatin-coated $60 \mathrm{~mm}$ tissue culture plates seeded with feeder cells (Cs irradiated C57BL/6J × 129S1/ SvImJ1 MEFs) prior to the start of reprograming with doxycycline. For all plates, ESC media was refreshed daily during reprograming

\section{AP staining and colony counting}

AP staining was performed directly on the $60-\mathrm{mm}$ plates using the Vector Red Alkaline Phosphatase Substrate Kit (Vector Laboratories, Burlingame, CA, USA) according to the manufacturer's directions. Both the number of 
AP-stained colonies and the total number of colonies on the plates were quantified using bright field microscopy at $100 \times$ magnification. Colonies were identified based on the following morphological criteria: well definedborder, three-dimensionality, and tightly packed cells. A grid system was used on the plates to facilitate colony counting. Each plate was counted twice and the mean number of AP-stained colonies and the mean total number of colonies was determined. The percentage of AP stained colonies was determined by dividing the mean number of AP-stained colonies by the mean total number of colonies and then multiplying by one hundred.

\section{Flow cytometric analysis}

Cells from the 6-well primary transformation plates were trypsinized, washed with PBS, fixed in $4 \%$ paraformaldehyde, washed again, and resuspended in blocking buffer (TBS buffer, $0.1 \%$ Triton X-100, and 1\% BSA) overnight at $4{ }^{\circ} \mathrm{C}$. The cell pellet was then washed, resuspended in unconjugated primary antibody for 1 hour at $4^{\circ} \mathrm{C}$, washed, and resuspended in a secondary fluorescent-conjugated antibody for an additional 1 hour at $4^{\circ} \mathrm{C}$. Cells were resuspended in blocking buffer and analyzed on a BD LSR II (Becton Dickinson Immunocytometry Systems, San Jose, CA, USA) flow cytometer and FACSDiva software (Becton Dickinson). Data were collected on $1 \times$ $10^{4}$ cells. Double staining with primary antibodies against SSEA1 (Millipore MAB4301, Billerica, MA, USA) and LIN28 (Abcam Inc. ab46020, Cambridge, MA, USA) with respective fluorescein isothiocyanate (FITC) (SouthernBiotech 1010-02, Birmingham, AL, USA) and PerCPCy5.5 (Santa Cruz Biotechnologies sc-45101, Santa Cruz, CA, USA) conjugated secondary antibodies was performed with resultant quadrant statistics including percentage of positive cells in each quadrant. Calibration of the flow cytometer and setting of gates was performed using non-transduced P2 MEFs as negative controls and established 1-A4 (C57BL/6J ×129S1/SvlmJ) iPSCs and v6.4 (C57BL/6J × 129S4/SvJae) ESCs (530 You,Y. 1998) as positive controls. The 1-A4 iPSC line was generated in our laboratory and validated via teratoma formation in NOD.CB17-Prkdc $c^{\text {scid } / J ~ m i c e ~ a n d ~ a b i l i t y ~ t o ~ g e n e r a t e ~ g e r m-~}$ line chimeras through blastocyst injection.

\section{iPSC line generation}

iPSC colonies from 6-well primary transformation plates were picked with pipette tips into individual wells of 96-well tissue culture plates containing trypsin. The trypsin was neutralized with DMEM and $10 \%$ FBS, and the cells within each well were then transferred to individual wells of 96-well tissue culture plates seeded with feeder cells in ESC media and expanded. Doxycycline was removed from the media at the 6-well plate stage (around P7) in order to establish doxycycline-independent cell lines from each strain. The cells were then further expanded (P10 to P15) in order to reach the cell numbers necessary for teratoma formation assays and for cryopreservation of stock from each strain.

\section{Teratoma formation and histological analysis}

iPSCs from one doxycycline-independent cell line from each strain were trypsinized, pelleted and suspended at $1 \times 10^{7}$ cells $/ \mathrm{mL}$ in MEF media, then $150 \mu \mathrm{l}$ of the cell suspension $\left(1.5 \times 10^{6}\right.$ cells $)$ was injected subcutaneously into the flank of a NOD.CB17-Prkdc scid/J mouse. For each strain, six injections were performed in 3 NOD.CB17Prkd $c^{\text {scid } / J ~ m i c e ~(b o t h ~ f l a n k s ~ o f ~ e a c h ~ m o u s e ~ w e r e ~ i n j e c t e d) . ~}$ Four to five weeks post-injection, tumors were surgically dissected, fixed in $4 \%$ paraformaldehyde, embedded in paraffin, sectioned, and stained with hematoxylin and eosin. All histologic sections were reviewed by a board-certified veterinary pathologist (TLS.) for teratoma formation.

\section{MEF proliferation assays}

Proliferation of non-transduced parent P2 MEFs from each strain was examined every 2 days over a total of 10 days. We seeded $1.9 \times 10^{5} \mathrm{MEFs}$ on each $60 \mathrm{~mm}$ tissue culture plate to be cultured and later harvested at the indicated time points to perform cell counts. MEFs were maintained in standard MEF media for the first 24 hours and then the media was changed to ESC media supplemented with doxycycline to simulate reprograming conditions for the remainder of the assay. Assays were performed using MEFs derived from two different embryos for each strain.

\section{Results and discussion}

\section{Strain differences in efficiency to generate iPSCs are} manifested in the early stages of reprograming

In order to assess potential strain background effects on iPSC generation during early reprograming, the primary transformation and $60 \mathrm{~mm}$ plates were evaluated for the total number of colonies, the percentage of colonies positive for AP staining, and the percentage of cells positive for SSEA1 and LIN28 expression. On both the 60-mm plates in which the cells were used for AP staining and colony counting and the 6-well primary transformation plates in which the cells were used for flow cytometric analysis, gross differences in the generation of iPSC colonies were observed such that cells had to be stained and counted, or harvested for flow cytometry, after only 8 days of reprograming in order to avoid overconfluency of cells from the most efficient strains (Figure 2A). This time point was much earlier than expected based on the doxycycline-inducible lentiviral reprograming system literature in which colonies are generally passaged or picked off of primary transformation plates around 13 to 21 days for expansion and/or evaluation [27-29], and 


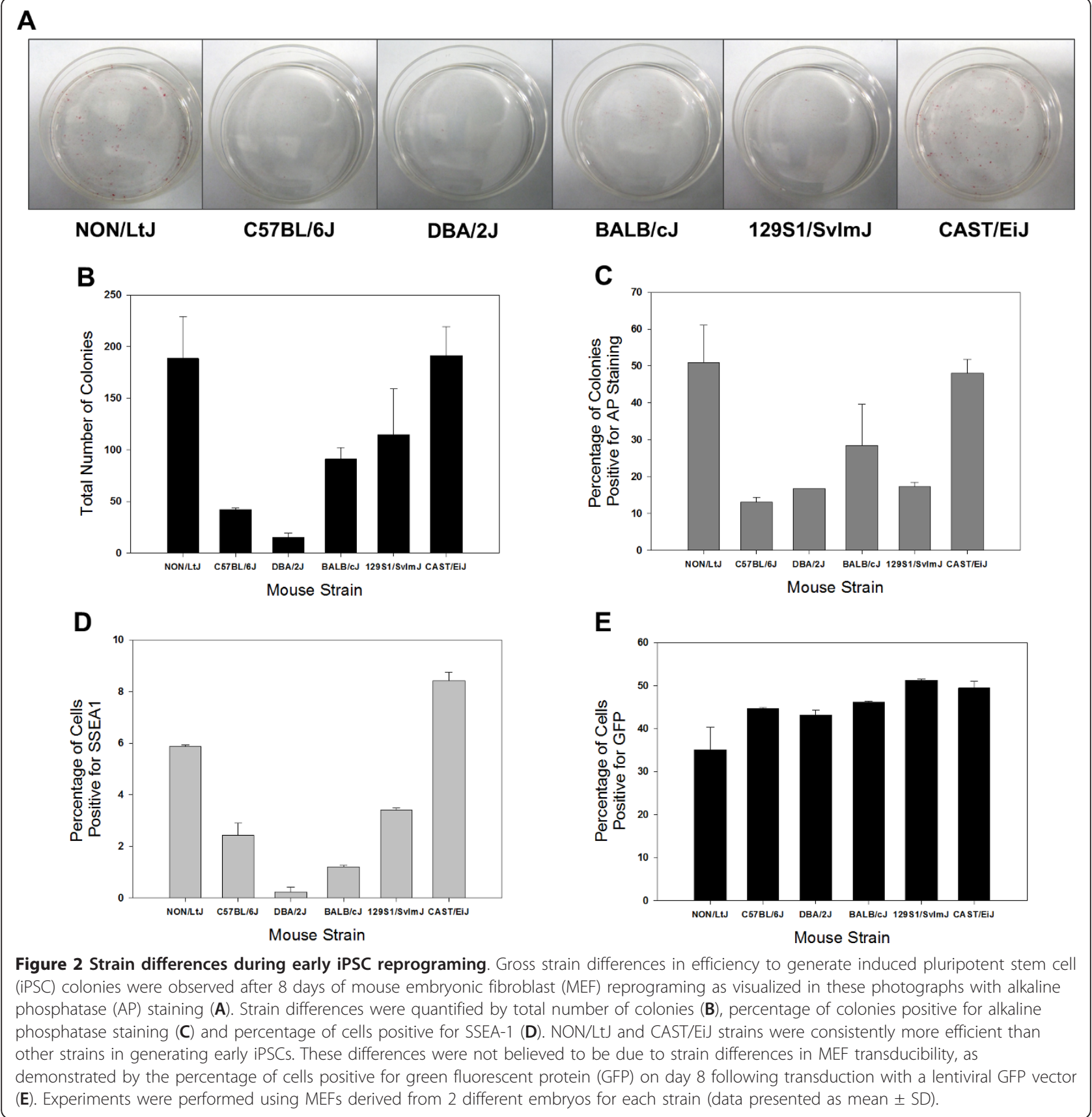

stresses the differences that can be observed when using strains of diverse genetic backgrounds. Because the iPSCs were harvested at this very early time point of 8 days, the resultant LIN28 expression was negative in the iPSCs from all six strains and only SSEA1 expression was included in the final analysis. This finding is consistent with the literature in which LIN28 is used as a marker for more established iPSCs and ESCs [30-32] as confirmed by our control iPSC (1-A4) and ESC (v6.4) lines.

During early reprograming, MEFs from all of the strains formed cell colonies exhibiting typical iPSC morphology that were AP-positive within 8 days after the start of reprograming. However, the total number of colonies and the percentage of AP-positive colonies varied dramatically between the strains. Notably, NON/LtJ and CAST/EiJ strains were more efficient than other strains (Figures 2B and 2C). Similarly, the percentage of cells positive for SSEA1 varied between the strains but paralleled the previous two parameters with NON/LtJ and CAST/EiJ having the highest percentage (Figure 2D). As expected, the percentage of cells positive for SSEA1 was low (between 0.11 and $8.64 \%$ ) at this early time point 
of 8 days after the start of reprograming for all strains. Using the same doxycycline-inducible lentiviral reprograming system, Brambrink et al. previously demonstrated that SSEA1 expression appears between 3 and 9 days of reprograming, whereas AP activity appears within 3 days of reprograming [27]. Brambrink et al. also showed that after 9 days of reprograming, about $7 \%$ of AP-positive cells were also SSEA1-positive [27]. This percentage of SSEA1-positive cells is consistent with our findings.

To ensure that the differences amongst strains in reprograming efficiency were not due to differences in lentiviral infection, P2 MEFs were seeded on 6-well plates at the same density as they were for reprograming, transduced with a lentiviral green fluorescent protein (GFP) vector (Addgene 14883, Cambridge, MA, USA) and maintained under reprograming conditions. After 8 days, the cells were trypsinized and the percentage of GFPpositive cells was determined using flow cytometry. The percentage of GFP-positive cells was very similar for all strains, ranging from $35.10 \pm 5.23 \%$ (mean $\pm \mathrm{SD}$ ) for NON/LtJ MEFs to $51.25 \pm 0.21 \%$ for 129 S1/SvImJ1 MEFs, suggesting that the strain differences in efficiency to generate iPSCs were not due to strain differences in MEF transducibilty (Figure 2E).

\section{Differences in proliferation of parent non-transduced MEFs correlate with differences in efficiency to generate iPSCs during early reprograming}

Proliferation of non-transduced parent P2 MEFs was examined every 2 days over a total of 10 days in order to determine if genetic differences in MEF proliferation could potentially be affecting the efficiency of iPSC generation (Figure 3A). Strain differences in MEF proliferation were observed over the 10 day period and a positive correlation was found between MEF growth rate and efficiency to generate iPSCs during early reprograming. This is demonstrated in Figure 3B where MEF growth rate and total number of colonies positive for AP staining are compared with a resultant $\mathrm{r}^{2}$ value of 0.75 . In particular, NON/LtJ and CAST/EiJ MEFs were the most proliferative and most efficient in generating iPSCs while DBA/2J MEFs were the least.

Interestingly, fibroblasts capable of increased proliferation through Trp53 deletion have increased iPSC generation efficiency [33-35]. It is possible that MEFs of the most efficient strains found in this study, NON/Lt and CAST/ EiJ, have a reduced rate of senescence compared to the other strains, which is allowing for more effective reprograming. The fact that the most proliferative MEFs were of the CAST/EiJ strain is also of interest as this wildderived inbred strain is the most genetically distinct strain that we examined.

The finding of this study that cellular proliferation rate is correlated with iPSC generation efficiency is consistent with those of Ruiz et al. in which the induction of cellular proliferation (through downregulation of $\mathrm{pRb}$ ) increased human iPSC reprograming efficiency [36]. In that study, Ruiz et al. also elegantly demonstrated that cell cycle arrest (through induction of the arrest inducers p15, p16, or $\mathrm{p} 21$ ) inhibits reprograming and actually drives iPSCs towards irreversible differentiation [36]. A potential
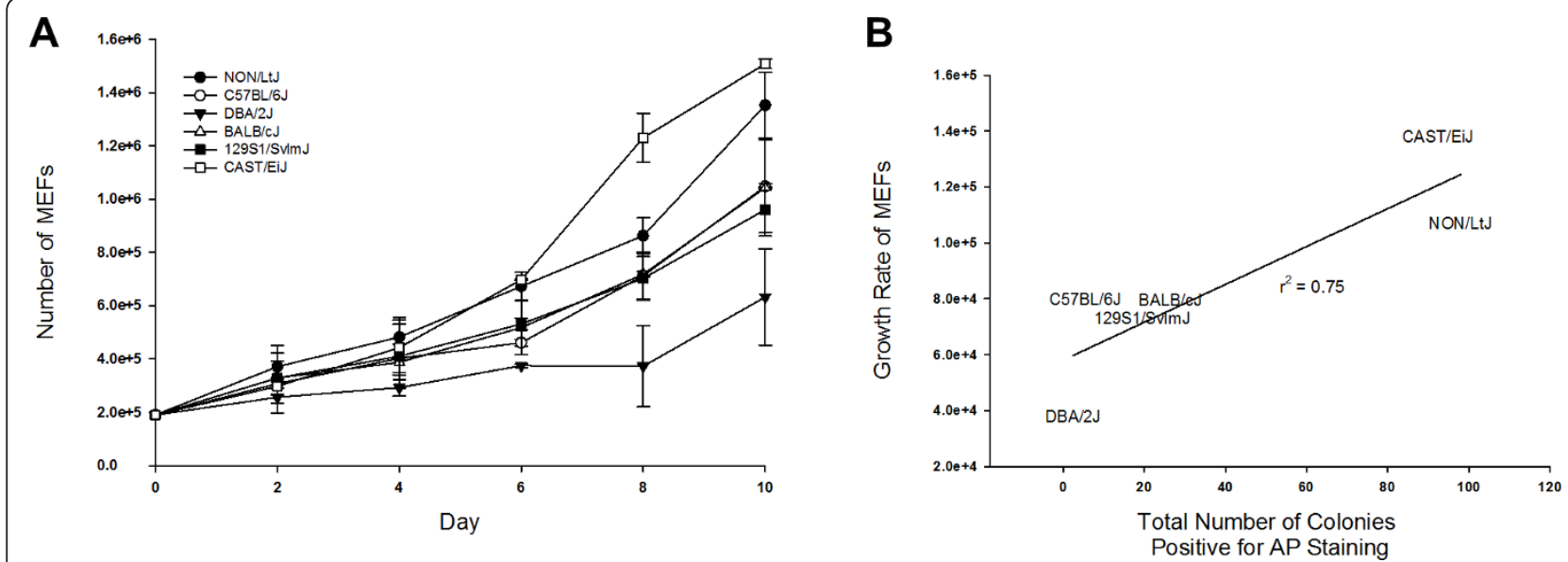

Figure 3 Strain differences in mouse embryonic fibroblast (MEF) proliferation. Strain differences in MEF proliferation were observed over 10 days and were well correlated with the observed strain differences in efficiency to generate induced pluripotent stem cells (iPSCs) during early reprograming. Non-transduced parent P3 MEFs from each strain were seeded at $1.9 \times 10^{5}$ cells per 60 -mm tissue culture plate on day 0 and then counted every 2 days over a total of 10 days (A). MEFs were maintained in standard MEF media for the first 24 hours after which the media was changed to embryonic stem cell (ESC) media supplemented with doxycycline to simulate reprograming conditions. MEFs derived from two different embryos were evaluated for each strain (data presented as mean \pm SD). The growth rate of the MEFs from each strain was determined from the slope of the linear regression curve fitted to the data set in $(\mathbf{A})$ for each strain. The growth rate of each strain was then plotted against the total number of colonies positive for alkaline phosphatase (AP) staining and a line of best fit determined, revealing a moderately strong positive correlation between iPSC generation efficiency and MEF proliferation as indicated by the $r^{2}$ value (B). 
follow-up study to this one in order to further elucidate the mechanisms behind the differences in genetic background effects on iPSC efficiency would be to alter the cellular proliferation of the MEFs for each strain, either through induction or arrest, and then examine the iPSC generation efficiency.

$B A L B / C J$ iPSCs require a higher concentration of LIF than other strains for cell line expansion and doxycycline independence

In order to determine if genetic background affects the pluripotent stability of iPSCs during later stages of reprograming, iPSC lines from all six strains were established and further expanded without doxycycline supplementation. Doxycycline-independent iPSC lines could be established using our standard conditions and ESC media for all strains except BALB/c), which were established only when supplemented with a higher concentration $(5 x)$ of leukemia inhibitory factor (LIF). This finding suggests that $\mathrm{BALB} / \mathrm{CJ}$ iPSCs may have reduced pluripotent stability and is consistent with the BALB/CJ ESC literature in which $\mathrm{BALB} / \mathrm{cJ}$ ESC lines were established only when using a $5 \times$ higher concentration of LIF than that needed for other strains [5-7]. The mechanism behind this requirement for

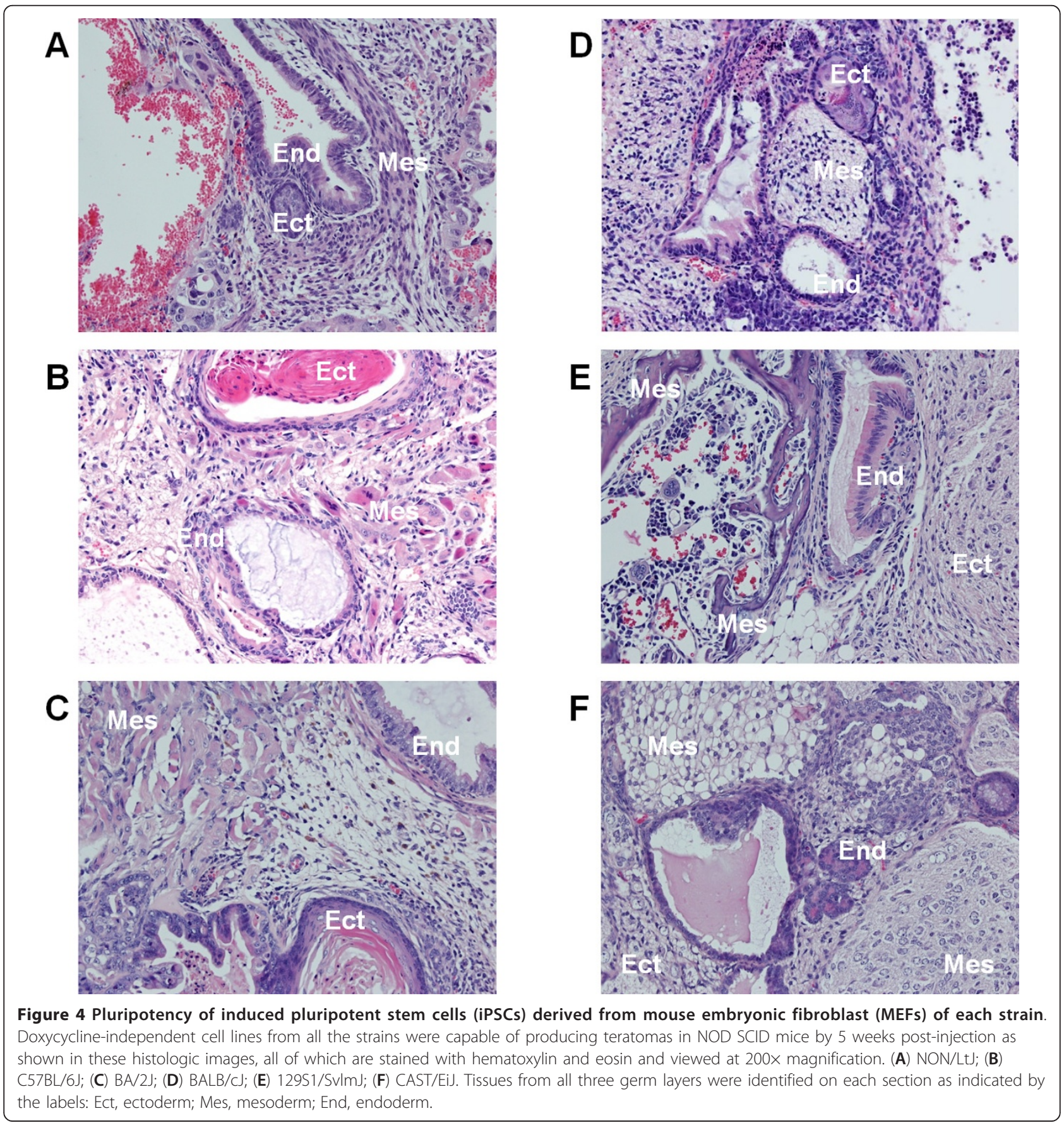


increased LIF supplementation in BALB/cJ cells has yet to be identified.

\section{Doxycycline-independent cell lines from all strains are capable of producing teratomas in SCID mice}

Doxycycline-independent cell lines from all the strains were capable of producing teratomas in NOD.CB17$P r k d c^{\text {scid } / J}$ mice by 5 weeks post injection, thereby demonstrating pluripotency (Figure 4). For all strains, the cell lines were between P10 and P15 and were the initial cell lines chosen for the teratoma assay. None of the cell lines from any of the strains required a repeat set of injections or the assay to be repeated with a different cell line.

iPSC lines in this study were not evaluated for their ability to generate chimeras or for germline competence, making direct comparison to the ESC literature on the effect of genetic background difficult beyond the finding of reduced pluripotent stability already discussed for the $\mathrm{BALB} / \mathrm{cJ}$ strain. While the $129 \mathrm{~S} 1 / \mathrm{Svlm}$ J strain was moderately efficient in generating IPSCs compared to the other strains in this study and the $129 \mathrm{~S} 1 /$ SvlmJ iPSC line was readily able to form teratomas in NOD.CB17$P r k d c^{s c i d} / \mathrm{J}$ mice, a conclusion cannot be drawn from these data as to whether or not this strain is as useful for generating iPSCs as it has been shown to be for generating ESCs for transgenic applications [6,7]. In addition, the two most efficient strains in this study, NON/ LtJ and CAST/EiJ, are strains that have not been examined for their ability to generate ESCs, making them intriguing candidates for future studies.

\section{Conclusions}

Our comparison of six different inbred mouse strains has revealed that genetic background does affect both the efficiency of generating iPSCs during the early stages of reprograming as well as the pluripotent stability of the cells during later stages of reprograming. These findings suggest that genetic background must be considered when interpreting results of iPSC studies in the literature and that iPSC derivation may need to be customized for different strains. In addition, our findings suggest that the proliferation rate of the fibroblasts is positively correlated with iPSC generation, suggesting a possible simple laboratory screening parameter to predict iPSC generation efficiency.

The two most efficient strains in this study, NON/Lt] and CAST/EiJ, may prove useful in the future for deriving iPSCs for transgenic purposes, as iPSCs from these strains appear to be robust. One limitation to this study, however, was that we did not evaluate the iPSCs lines for their ability to generate chimeras and for germline competence. This information is essential for ultimately determining which strain may be most beneficial for transgenic applications.
In conclusion, we have shown that there are strain differences in efficiency to generate iPSCs during the early stages of reprograming and that these strain differences are correlated with those observed in proliferation of parent MEFs. We have also shown that there are strain differences in pluripotent stability as far as ability to expand iPSC lines and achieve doxycycline independence. These findings have important implications both for future iPSC applications as well as for future investigation into determining the genes responsible for reprograming efficiency and stability. It is possible that the Collaborative Cross, of which three of the strains examined in this study are progenitors, could be used to identify such genes.

\section{Abbreviations}

AP: alkaline phosphatase; BSA: bovine serum albumin; CMV: cytomegalovirus DMEM: Dulbecco's modified eagle's medium; DMSO: dimethyl sulfoxide; ESC: embryonic stem cell; FITC: fluorescein isothiocyanate; GFP: green fluorescent protein; iPSC: induced pluripotent stem cell; LIF: leukemia inhibitory factor; MEF: mouse embryonic fibroblast; P2: passage 2; PBS: phosphate buffered saline; SSEA1: stage-specific embryonic antigen 1; TetO: tetracycline operator; TTF: tail tip fibroblast.

\section{Acknowledgements}

This work was supported by Empire State Stem Cell Fund contract \#C024400 (LAF and JCS) and National Institutes of Health grant \#1 K08AR060875-01A1 (LVS). The authors would like to thank Mr. Robert Munroe for his help with animal care and for his contributions to the experimental design. The authors would also like to acknowledge use of the Cornell University Irradiator Core Facility and support from National Center for Research Resources grant \#S10RR023781.

\section{Author details}

'Department of Clinical Sciences, College of Veterinary Medicine, Cornell University, Ithaca, NY 14853, USA. ²Department of Biomedical Sciences, College of Veterinary Medicine, Cornell University, Ithaca, NY 14853, USA.

\section{Authors' contributions}

LVS, CMA, JCS, and LAF designed the study. LVS and CMA performed the experiments. LVS performed tissue collections (for teratoma assays), on which LVS and TLS carried out histologic assessments. All authors contributed to data analysis and interpretation. LVS, JCS, and LAF were responsible for drafting the manuscript. All authors revised the manuscript and approved the final version.

\section{Authors' information}

Author details

1) Lauren V Schnabel, VMC C3-105, College of Veterinary Medicine, Cornell University, Ithaca, NY 14853, USA. Email: Ivs3@cornell.edu

2) Christian M Abratte, VRT T9-010, College of Veterinary Medicine, Cornell University, Ithaca, NY 14853, USA. Email: ca258@cornell.edu

3) John C Schimenti, VRT T9-014A, College of Veterinary Medicine, Cornell University, Ithaca, NY 14853, USA. Email: jcs92@cornell.edu

4) Teresa L Southard, Diagnostic Lab North, Room A1-212, Cornell University, Ithaca, NY 14853, USA. Email: tls93@cornell.edu

5) Lisa A Fortier, VMC C3-181, College of Veterinary Medicine, Cornell University, Ithaca, NY 14853, USA. Email: laf4@cornell.edu. §Corresponding author.

\section{Competing interests}

The authors declare that they have no competing interests. 


\section{References}

1. Okita K, Matsumura Y, Sato Y, Okada A, Morizane A, S Okamoto, Hong H, Nakagawa M, Tanabe K, Tezuka K, Shibata T, Kunisada T, Takahashi M, Takahashi J, Saji H, Yamanaka S: A more efficient method to generate integration-free human iPS cells. Nat Methods 2011, 8:409-412.

2. Okita K, Yamanaka S: Induced pluripotent stem cells: opportunities and challenges. Philos Trans R Soc Lond B Biol Sci 2011, 366:2198-2207.

3. Puri MC, Nagy A: Concise Review: ES vs. iPS Cells; the Game is on. Stem Cells 2011, 30:10-14

4. Chun YS, Byun K, Lee B: Induced pluripotent stem cells and personalized medicine: current progress and future perspectives. Anat Cell Biol 2011, 44:245-255.

5. Baharvand $\mathrm{H}$, Matthaei KI: Culture condition difference for establishment of new embryonic stem cell lines from the C57BL/6 and BALB/c mouse strains. Vitro Cell Dev Biol Anim 2004, 40:76-81

6. Kawase E, Suemori H, Takahashi N, Okazaki K, Hashimoto K, Nakatsuji N: Strain difference in establishment of mouse embryonic stem (ES) cell lines. Int J Dev Biol 1994, 38:385-390.

7. Suzuki O, Matsuda J, Takano K, Yamamoto Y, Asano T, Naiki M, Kusanagi M: Effect of genetic background on establishment of mouse embryonic stem cells. Exp Anim 1999, 48:213-216.

8. lijima S, Tanimoto Y, Mizuno S, Daitoku Y, Kunita S, Sugiyama F, Yagami K: Effect of different culture conditions on establishment of embryonic stem cells from BALB/CAJ and NZB/BINJ mice. Cell Reprogram 2010, 12:679-688.

9. Wernig M, Meissner A, Foreman R, Brambrink T, Ku M, Hochedlinger K, Bernstein $B E$, Jaenisch $R$ : In vitro reprograming of fibroblasts into a pluripotent ES-cell-like state. Nature 2007, 448:318-324.

10. Maherali N, Sridharan R, Xie W, Utikal J, Eminli S, Arnold K, Stadtfeld M, Yachechko R, Tchieu J, Jaenisch R, Plath K, Hochedlinger K: Directly reprogrammed fibroblasts show global epigenetic remodeling and widespread tissue contribution. Cell Stem Cell 2007, 1:55-70.

11. Takahashi K, Yamanaka S: Induction of pluripotent stem cells from mouse embryonic and adult fibroblast cultures by defined factors. Cell 2006, 126:663-676.

12. Okita K, Nakagawa M, Hyenjong H, Ichisaka T, Yamanaka S: Generation of mouse induced pluripotent stem cells without viral vectors. Science 2008, 322:949-953.

13. Okita K, Ichisaka T, Yamanaka S: Generation of germline-competent induced pluripotent stem cells. Nature 2007, 448:313-317.

14. Li Z, Yang CS, Nakashima K, Rana TM: Small RNA-mediated regulation of iPS cell generation. EMBO J 2011, 30:823-834.

15. Fujiwara M, Yan P. Otsuji TG, Narazaki G, Uosaki H, Fukushima H, Kuwahara K, Harada M, Matsuda H, Matsuoka S, Okita K, Takahashi K, Nakagawa M, Ikeda T, Sakata R, Mummery CL, Nakatsuji N, Yamanaka S, Nakao K, Yamashita JK: Induction and enhancement of cardiac cell differentiation from mouse and human induced pluripotent stem cells with cyclosporin-A. PLoS One 2011, 6:e16734.

16. Hayashi K, Ohta H, Kurimoto K, Aramaki S, Saitou M: Reconstitution of the mouse germ cell specification pathway in culture by pluripotent stem cells. Cell 2011, 146:519-532.

17. Hanna J, Markoulaki S, Mitalipova M, Cheng AW, Cassady JP, Staerk J, Carey BW, Lengner CJ, Foreman R, Love J, Gao Q, Kim J, Jaenisch R: Metastable pluripotent states in NOD-mouse-derived ESCs. Cell Stem Cell 2009, 4:513-524.

18. Takata Y, Kishine H, Sone T, Andoh T, Nozaki M, Poderycki M, Chesnut JD, Imamoto F: Generation of iPS Cells Using a BacMam Multigene Expression System. Cell Struct Funct 2011, 36:209-222.

19. Liu J, Ashton MP, Sumer H, O'Bryan MK, Brodnicki TC, Verma PJ: Generation of stable pluripotent stem cells from NOD mouse tail-tip fibroblasts. Diabetes 2011, 60:1393-1398.

20. Marson A, Foreman R, Chevalier B, Bilodeau S, Kahn M, Young RA, Jaenisch R: Wnt signaling promotes reprograming of somatic cells to pluripotency. Cell Stem Cell 2008, 3:132-135.

21. Lyssiotis CA, Foreman RK, Staerk J, Garcia M, Mathur D, Markoulaki S, Hanna J, Lairson LL, Charette BD, Bouchez LC, Bollong M, Kunick C, Brinker A, Cho CY, Schultz PG, Jaenisch R: Reprograming of murine fibroblasts to induced pluripotent stem cells with chemical complementation of Klf4. Proc Natl Acad Sci USA 2009, 106:8912-8917.

22. Petkov PM, Ding Y, Cassell MA, Zhang W, Wagner G, Sargent EE, Asquith $S$, Crew V, Johnson KA, Robinson P, Scott VE, Wiles MV: An efficient SNP system for mouse genome scanning and elucidating strain relationships. Genome Res 2004, 14:1806-1811.

23. Yang H, Bell TA, Churchill GA, Pardo-Manuel de Villena F: On the subspecific origin of the laboratory mouse. Nat Genet 2007, 39:1100-1107.

24. Keane TM, Goodstadt L, Danecek P, White MA, Wong K, Yalcin B, Heger A, Agam A, Slater G, Goodson M, Furlotte NA, Eskin E, Nellaker C, Whitley H, Cleak J, Janowitz D, Hernandez-Pliego P, Edwards A, Belgard TG, Oliver PL, McIntyre RE, Bhomra A, Nicod J, Gan X, Yuan W, van der Weyden L, Steward CA, Bala S, Stalker J, Mott R, Durbin R, Jackson IJ, Czechanski A, Guerra-Assuncao JA, Donahue LR, Reinholdt LG, Payseur BA, Ponting CP, Birney E, Flint J, Adams DJ: Mouse genomic variation and its effect on phenotypes and gene regulation. Nature 2011, 477:289-294.

25. Chesler EJ, Miller DR, Branstetter LR, Galloway LD, Jackson BL, Philip VM, Voy BH, Culiat CT, Threadgill DW, Williams RW, Churchill GA, Johnson DK, Manly KF: The Collaborative Cross at Oak Ridge National Laboratory: developing a powerful resource for systems genetics. Mamm Genome 2008, 19:382-389.

26. Threadgill DW, Miller DR, Churchill GA, de Villena FP: The collaborative cross: a recombinant inbred mouse population for the systems genetic era. ILAR J 2011, 52:24-31.

27. Brambrink T, Foreman R, Welstead GG, Lengner CJ, Wernig M, Suh $H$, Jaenisch R: Sequential expression of pluripotency markers during direct reprograming of mouse somatic cells. Cell Stem Cell 2008, 2:151-159.

28. Welstead GG, Brambrink T, Jaenisch R: Generating iPS cells from MEFS through forced expression of Sox-2, Oct-4, c-Myc, and Klf4. J Vis Exp 2008, , 14: 734, doi:10.3791/734

29. Chuang $\mathrm{CH}$, Wallace MD, Abratte C, Southard T, Schimenti JC: Incremental genetic perturbations to MCM2-7 expression and subcellular distribution reveal exquisite sensitivity of mice to DNA replication stress. PLoS Genet 2010, 6:e1001110.

30. Kim EY, Jeon K, Park HY, Han YJ, Yang BC, Park SB, Chung HM, Park SP: Differences between cellular and molecular profiles of induced pluripotent stem cells generated from mouse embryonic fibroblasts. Cell Reprogram 2010, 12:627-639.

31. Cai J, Chen J, Liu Y, Miura T, Luo Y, Loring JF, Freed WJ, Rao MS, Zeng X: Assessing self-renewal and differentiation in human embryonic stem cell lines. Stem Cells 2006, 24:516-530.

32. Richards M, Tan SP, Tan JH, Chan WK, Bongso A: The transcriptome profile of human embryonic stem cells as defined by SAGE. Stem Cells 2004, 22:51-64.

33. Hong H, Takahashi K, Ichisaka T, Aoi T, Kanagawa O, Nakagawa M, Okita K, Yamanaka S: Suppression of induced pluripotent stem cell generation by the p53-p21 pathway. Nature 2009, 460:1132-1135.

34. Kawamura T, Suzuki J, Wang W, Menendez S, Morera LB, Raya A, Wahl GM, Izpisua Belmonte JC: Linking the p53 tumour suppressor pathway to somatic cell reprograming. Nature 2009, 460:1140-1144.

35. Menendez S, Camus S, Izpisua Belmonte JC: P53: Guardian of reprograming. Cell Cycle 2010, 9:3887-3891.

36. Ruiz S, Panopoulos AD, Herrerias A, Bissig K, Lutz M, Berggren WT, Verma IM, Belmonte $\mathrm{JCl}$ : A high proliferation rate is required for cell reprograming and maintenance of human embryonic stem cell identity. Curr Biol 2011, 21:45-52.

doi:10.1186/scrt121

Cite this article as: Schnabel et al:: Genetic background affects induced pluripotent stem cell generation. Stem Cell Research \& Therapy 2012 3:30. 